\title{
PROPELENTE SÓLIDO COMPÓSITO POLIBUTADIÊNICO: I- INFLUÊNCIA DO AGENTE DE LIGAÇÃO
}

\author{
Jairo Sciamareli, Marta Ferreira Koyama Takahashi e José Maria Teixeira
}

Divisão de Química, Instituto de Aeronáutica e Espaço, Centro Técnico Aeroespacial, São José dos Campos - SP

Koshun Iha*

Depto. de Química, Instituto Tecnológico de Aeronáutica, Centro Técnico Aeroespacial, 12220-450 São José dos Campos - SP

Recebido em 20/10/00; aceito em 27/6/01

\begin{abstract}
SOLID POLYURETHANE-BASED COMPOSITE PROPELLANT: I- INFLUENCE OF THE BONDING AGENT. In the Brazilian Complete Spacial Mission, the research of Vehicle Satellite Launcher is important. The solid propellant composite used in this vehicle is made with ammonium perchlorate, aluminiun powder and hydroxy terminated polibutadiene resin. In the propellant the bonding agent has a function to promote the interaction between solids and polimeric matrix, improving mechanical properties as stress, strain, aging characteristics and moisture embrittlement. Due its importance in propellant, bonding agent is consider as industrial secret and the literature about it is generic, without greater informations.
\end{abstract}

Keywords: propellants; bonding agents; influence in mechanical properties.

\section{INTRODUÇÃO}

Propulsores de veículos espaciais, também conhecidos como motores-foguetes, exigem propelentes especiais, altamente energéticos, que ofereçam alto impulso específico. Um motor-foguete movido a propelente sólido é constituído basicamente de um tubo metálico cilíndrico, denominado envelope motor, preenchido quase totalmente com massa propelente, o grão propelente.

Propelente sólido é uma mistura complexa e estável de compostos redutores e oxidantes que, quando ignitados, queimam de uma maneira homogênea, contínua e controlada, formando a altas temperaturas moléculas gasosas de baixa massa molecular. Desta forma, ocorre na câmara do motor-foguete, um formidável aumento de espécies gasosas com grande elevação de temperatura e pressão no sistema. $\mathrm{Na}$ saída do motor-foguete existe um estreitamento, chamado garganta de exaustão ou tubeira, por onde os gases resultantes da queima saem acelerados. O aumento de pressão, que pode atingir em certos casos $100 \mathrm{Mpa}$, resulta no impulsionamento do foguete ${ }^{1}$.

No motor-foguete existe, entre o envelope motor e o propelente, uma proteção térmica constituída por uma fina camada de borracha e um adesivo denominado "liner", com a finalidade de não permitir que a alta temperatura de combustão do propelente danifique a estrutura do foguete. Na Figura 1 está o esquema simplificado de um motor-foguete.

\section{COMPOSIÇÃO TÍPICA DE UM PROPELENTE SÓLIDO COMPÓSITO}

Os propelentes sólidos, do tipo compósito, são constituídos de três componentes principais: Uma porção orgânica, rica em carbono e hidrogênio, conhecida como binder, que serve como ligante e gerador de gases; um sal inorgânico oxidante, rico em oxigênio, e um auxiliar balístico, normalmente um metal, tendo os sólidos uma micro granulometria bem definida ${ }^{2,3}$.

No propelente compósito o binder é a matriz elastomérica que além de conter, incorpora as partículas sólidas do metal combustível e

*e-mail: koshun@ief.ita.cta.br

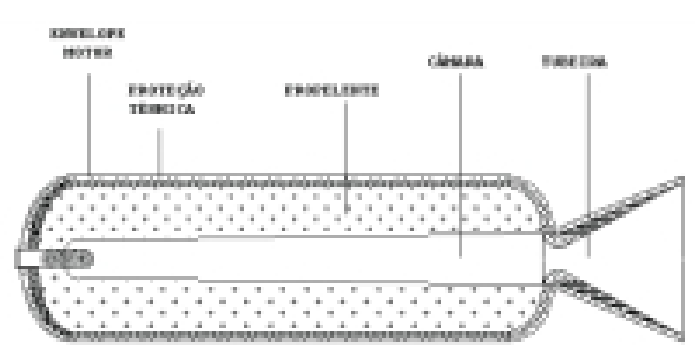

Figura 1. Esquema simplificado de um motor-foguete

do oxidante. Sendo a fase contínua do propelente, o binder tem múltiplas funções pois, além de ser a principal fonte de carbono e hidrogênio do propelente, deve manter unidas as partículas do metal e do oxidante, formando uma consistente massa elástica capaz de resistir às solicitações provocadas pelas tensões mecânicas e térmica. Portanto, o binder também contribui para as propriedades mecânicas do propelente. E é importante que tenha afinidade com as partes constituintes do motor-foguete, proteção térmica e componentes metálicos ${ }^{2}$.

Os primeiros compostos poliméricos a serem utilizados como ligantes foram o betumem, o PVC e o poliisobutileno que se mostraram inadequados, por fornecerem propelentes com propriedades mecânicas insatisfatórias. Desenvolveu-se posteriormente, na década de 50, polímeros líquidos que podiam ser curados através de ligações cruzadas após a adição da carga do propelente. Os primeiros destes polímeros foram os polissulfetos e poliéster. Entretanto, os grupos que mais se destacaram e são atualmente os mais utilizados são as poliuretanas e os polibutadienos.

$\mathrm{O}$ oxidante é a fonte de oxigênio que, de maneira exotérmica, oxida as partículas sólidas e metálicas gerando os gases propulsivos. Um dos primeiros sais a ser utilizado foi o perclorato de potássio $\left(\mathrm{KClO}_{4}\right)$, que apresentava o inconveniente de não se transformar totalmente em gases durante a combustão do propelente. Foi substituído pelo nitrato de amônio $\left(\mathrm{NH}_{4} \mathrm{NO}_{3}\right)$. Este por ser muito higroscópico, acarretava problemas ao propelente. Atualmente, o oxidante mais utilizado é o perclorato de amônio $\left(\mathrm{NH}_{4} \mathrm{ClO}_{4}\right)$. Este sal além de ser pouco higroscópico, ter boa estabilidade química e alto teor de oxigênio, se transforma totalmente em gases durante a combustão produzindo sistemas mais energéticos. 
Os auxiliares balísticos são introduzidos na formulação com a finalidade de aumentar as propriedades energéticas do propelente. Regra geral, são elementos metálicos. O metal mais comumente utilizado é o alumínio em pó com forma esférica. Outros metais como magnésio e zircônio podem ser utilizados, porém, normalmente com resultados inferiores ao alumínio. O berílio apresenta os melhores resultados, porém não pode ser utilizado devido à formação de óxidos altamente tóxicos no processo de combustão ${ }^{4}$. Além destes três componentes básicos, são ainda incorporados ao propelente os chamados aditivos, compostos presentes em pequenas quantidades mas, que muito contribuem para melhorar a processabilidade e as propriedades mecânicas do propelente. São plastificantes, agentes de cura, agentes de ligação, catalisadores de cura e de queima, antioxidantes e agentes de processamento.

O plastificante tem como importante função melhorar as propriedades mecânicas do propelente, tais como fluidez, flexão, dureza, alongamento e resistência à tração. Alguns plastificantes utilizados são, entre outros, o Dioctilazelato (DOZ), Dioctilftalato (DOP), dibutilftalato (DBF), Dioctilsebacato (DOS) e Isodecilpelargonato (IDP). O único inconveniente destes plastificantes é que geralmente, devido ao fato de não estarem amarrados à estrutura da cadeia polimérica, tendem a migrar pelo propelente sólido arrastando seus constituintes ${ }^{5}$.

O agente de cura tem como função promover a formação de ligações cruzadas já que a massa do propelente, com todos os seus constituintes, é líquida sendo, portanto, necessário a presença de um agente endurecedor, um reticulante, para que ocorra a cura da massa do propelente, ou seja, sua solidificação. Os agentes de cura mais utilizados são: Tolueno diisocianato (TDI), Isoforona diisocianato (IPDI), Hexametileno diisocianato (HMDI), p-Fenileno diisocianato (PDI), 1,5-Naftaleno diisocianato (NDI), Metileno-bis(p-fenil-isocianato) (MDI), entre outros.

$\mathrm{O}$ agente de ligação tem a importante função de promover a interação entre a carga sólida com o binder, ligando-os, não permitindo que as partículas do primeiro fiquem "soltas" na matriz polimérica, o que acarreta perda de qualidade nas propriedades mecânicas.

Outros componentes importantes do propelente são o antioxidante que tem como função evitar o envelhecimento do propelente, principalmente a oxidação da resina pela quebra das duplas ligações, com consequente aumento da densidade de ligações cruzadas na cadeia polimérica $^{5}$; o agente de processamento, que é quimicamente inerte e tem como função diminuir a viscosidade da massa, deixando-a mais fluída, geralmente um silicone, lecitina de soja ou um sorbitato e por fim, o catalisador de queima, normalmente um óxido metálico, como por exemplo, $\mathrm{Fe}_{2} \mathrm{O}_{3}, \mathrm{NiO}, \mathrm{CoO},\left(\mathrm{Cr}_{2} \mathrm{O}_{3}\right)_{x}(\mathrm{CuO})$ y, que tem a função de acelerar a velocidade de queima de acordo com parâmetros de propulsão pre-estabelecidos. Convém ressaltar que os aditivos participam, em geral, da combustão do propelente fazendo o mesmo papel do binder, o de combustível.

\section{PROPELENTE SOB ASPECTO QUÍMICO}

O Centro Técnico Aeroespacial, CTA, tem, homologado, um propelente típico utilizado nos foguetes tipo Sonda e no Veículo Lançador de Satélites, VLS. Este propelente é do tipo compósito poliuretânico, que utiliza alumínio, perclorato de amônio e resina polibutadiênica hidroxilada e queima, basicamente, por meio de uma reação de óxido-redução ${ }^{6}$.

$\mathrm{NH}_{4} \mathrm{ClO}_{4}+\left(\mathrm{CH}_{2}\right)_{\mathrm{n}}+2 \mathrm{Al} \rightarrow 1 / 2 \mathrm{~N}_{2}+\mathrm{CO}+5 / 2 \mathrm{H}_{2}+\mathrm{Al}_{2} \mathrm{O}_{3}+\mathrm{HCl}$

Na verdade, a química de ignição e combustão dos propelentes baseados no sal oxidante perclorato de amônio envolve processos complexos governados por vários fatores tais como características do oxidante, do binder, pressão, concentração do oxidante, reações de pré-ignição entre outros ${ }^{7}$. No geral, as reações que ocorrem entre o oxidante e o binder ainda não estão completamente esclarecidas e é muito difícil a partir de qualquer modelo fornecer uma completa descrição do processo de combustão . $^{8}$.

Os principais constituintes do propelente são o perclorato de amônio, alumínio em pó e resina. Estes três constituintes correspondem aproximadamente a $95 \%$ da massa do propelente. Os outros 5\% são aditivos, englobando um plastificante, agente de ligação, antioxidante, catalisador de cura, agente de cura, anti catalisador de cura e agente de processamento, sendo o plastificante cerca de $60 \%$ da massa dos aditivos. A proporção de cada um destes constituíntes pode variar de acordo com parâmetros pré-estabelecidos, por exemplo, o oxidante varia de um mínimo de $60 \%$ a um máximo de $90 \%$ da massa total de propelente. Outros, como o catalisador de queima e o agente de processamento, podem ou não ser utilizados.

Como já foi mencionado, as reações que ocorrem são do tipo oxiredução, onde o sal perclorato de amônio é o agente oxidante e os outros componentes funcionam como agentes redutores. Praticamente toda energia fornecida provém da reação do perclorato de amônio com o alumínio em pó e a resina hidroxilada. Tanto o perclorato como o alumínio devem ser finamente divididos aumentando suas áreas específicas e assim, melhorando a superfície de contato.

A resina consiste de um polibutadieno líquido com terminações hidroxiladas. As insaturações servem como fonte de energia e as terminações hidroxila promovem a reação de formação de grupos uretana ao reagir com um isocianato. A resina ainda funciona como um veículo para homogenizar os sólidos, perclorato e alumínio, através de uma maceração, resultando em uma massa líquida e viscosa. A mistura líquida é transferida para o tubo do motor foguete, equipado com um mandril central que irá dar a configuração interna ao propelente. Esta massa sofre endurecimento à medida que a reação de cura (formação do poliuretano) se processa sob temperatura controla$\mathrm{da}$, de acordo com as características do projeto.

O plastificante utilizado é o dioctilazelato $(\mathrm{DOZ})$ e, como já mencionado, tem a função de melhorar as propriedades mecânicas do propelente como fluidez, flexão, dureza, alongamento e resistência à tração.

O agente de cura utilizado para promover a reticulação e posterior solidificação da massa do propelente é o isoforona diisocianato (IPDI). Este, através do grupo isocianato $(-\mathrm{N}=\mathrm{C}=\mathrm{O})$, reage com os grupos $\mathrm{OH}$ da resina resultando em grupos uretânicos, promovendo a formação da matriz polimérica que envolve e aglomera todos os constituintes do propelente.

$$
(\mathrm{O}=\mathrm{C}=\mathrm{N}-\mathrm{R})_{\mathrm{n}}+\left(\mathrm{R}^{\prime}-\mathrm{OH}\right)_{\mathrm{n}} \rightarrow\left(\mathrm{R}^{\prime}-\mathrm{O}-\mathrm{CO}-\mathrm{NH}-\mathrm{R}\right)_{\mathrm{n}}
$$

Em sistemas poliuretânicos são empregados como catalisadores de cura alguns compostos complexos organometálicos e aminas terciárias. As aminas atuam também como agentes de reticulação aumentando a densidade de ligações cruzadas da matriz polimérica. Sabe-se que a atividade catalítica dos acetilacetonatos metálicos é efetivo na reação da hidroxila alcoólica com isocianato. Entre os acetilacetonatos, o acetilacetonato férrico, $\left[\mathrm{Fe}(\mathrm{AA})_{3}\right]$ é o utilizado. Um possível mecanismo para esta reação é aqui apresentado9:

$$
\mathrm{Fe}(\mathrm{AA})_{3}+\mathrm{ROH} \rightarrow \mathrm{Fe}(\mathrm{AA})_{2} \mathrm{OR}+\mathrm{HAA}
$$

$$
\mathrm{Fe}(\mathrm{AA})_{2} \mathrm{OR}+\mathrm{ROH} \leftrightarrow\left[\mathrm{Fe}(\mathrm{AA})_{2}(\mathrm{OR})_{2}\right] \mathrm{H}
$$

$\left[\mathrm{Fe}(\mathrm{AA})_{2}(\mathrm{OR})_{2}\right] \mathrm{H}+\mathrm{RNCO} \rightarrow \mathrm{RNHCOOR}+\mathrm{Fe}(\mathrm{AA})_{2} \mathrm{OR}$ 
Porém, seu efeito catalítico é muito intenso, aumentando rapidamente a viscosidade da massa, não possibilitando um tempo de trabalho (pot-life) adequado às condições de carregamento do motorfoguete, ou seja, a massa do propelente torna-se muito viscosa a ponto de não escorrer ao carregar o motor-foguete. A redução da concentração do catalisador não é recomendada, uma vez que esse procedimento prejudicaria a reação da hidroxila com isocianato e outras reações secundárias poderiam acontecer como, por exemplo, com a umidade.

$$
\mathrm{R}-\mathrm{N}=\mathrm{C}=\mathrm{O}+\mathrm{H}_{2} \mathrm{O} \rightarrow[\mathrm{R}-\mathrm{NH}-\mathrm{COOH}] \rightarrow \mathrm{R}-\mathrm{NH}_{2}+\mathrm{CO}_{2}
$$

Esta amina primária é mais reativa que as hidroxilas alcoólicas e dá origem a outra reação indesejável, a formação de uréia simétrica, a qual pode reagir com outro grupo isocianato resultando em um propelente com alta dureza devido à formação de biuretos ${ }^{10}$.

$$
\begin{gathered}
\mathrm{R}-\mathrm{N}=\mathrm{C}=\mathrm{O}+\mathrm{H}_{2} \mathrm{~N}-\mathrm{R}^{\prime} \rightarrow \mathrm{R}-\mathrm{NH}-\mathrm{CO}-\mathrm{NH}-\mathrm{R}{ }^{\prime} \\
\mathrm{R}-\mathrm{N}=\mathrm{C}=\mathrm{O}+\mathrm{R}^{\prime}-\mathrm{NH}-\mathrm{CO}-\mathrm{NH}-\mathrm{R}{ }^{\prime} \rightarrow \mathrm{R} \text { '-N(CONHR)CONH-R"}
\end{gathered}
$$

Sabe-se, também, que a adição de pequena quantidade de acetilacetona (HAA) reduz o efeito catalítico do acetilacetonato férrico, o qual é restaurado à medida que o acetilacetona é consumido pelo alumínio ${ }^{11}$.

$$
3 \mathrm{CH}_{3} \mathrm{COCH}_{2} \mathrm{COCH}_{3}+\mathrm{Al} \rightarrow \mathrm{Al}\left(\mathrm{CH}_{3} \mathrm{COCHCOCH}_{3}\right)_{3}+3 / 2 \mathrm{H}_{2}
$$

\section{A IMPORTÂNCIA DO AGENTE DE LIGAÇÃO}

Em um suposto propelente fabricado sem agente de ligação, a fraca interação entre o cristal do sal oxidante e a massa polimérica resulta em um propelente com propriedades mecânicas deficientes. Este propelente ao sofrer tensões térmica (por exemplo, contração e expansão térmica) e/ou mecânicas (por exemplo, encolhimento durante a polimerização), propicia o surgimento de vacúolos, pequenas bolsas vazias na parte polimérica, em torno das partículas sólidas. Os vacúolos, posteriormente atingem a superfície da partícula sólida descolando-a da massa polimérica, a qual vai esgarçando-se, formando um vacúolo maior que, agora, passa a conter em seu interior a partícula sólida solta ${ }^{1,12}$. A Figura 2 mostra a ocorrência deste fenômeno em um propelente tensionado. Estes vazios se propagam, expandindo com o aumento da tensão. Isto causa um enfraquecimento das propriedades mecânicas, além de permitir a presença de umidade no propelente.

A presença de umidade pode ocasionar a dissolução da superfície da partícula oxidante, com a formação de ácido, atacando o polímero, não permitindo, assim, que este propelente seja estocado e armazenado por longo tempo.

$$
\begin{gathered}
\mathrm{NH}_{4} \mathrm{ClO}_{4}+\mathrm{H}_{2} \mathrm{O} \rightarrow \mathrm{HClO}_{4}+\mathrm{NH}_{4} \mathrm{OH} \\
\mathrm{NH}_{4} \mathrm{OH} \rightarrow \mathrm{NH}_{3}+\mathrm{H}_{2} \mathrm{O}
\end{gathered}
$$

Durante a queima do propelente outros problemas podem ocorrer a partir deste fenômeno. Os vacúolos e as partículas sólidas soltas agem como pontos de rachadura, permitindo um aumento da área superficial de queima e a possibilidade da chama se propagar abaixo da superfície de queima. A soma destes fatores causa maior velocidade de queima do propelente, acarretando um aumento repentino na produção de gases de combustão e maior pressurização na câmara de combustão. Como conseqüência, pode ocorrer falha nas especificações do motor-foguete, resultando então, em sua explosão ${ }^{1,13-15}$.

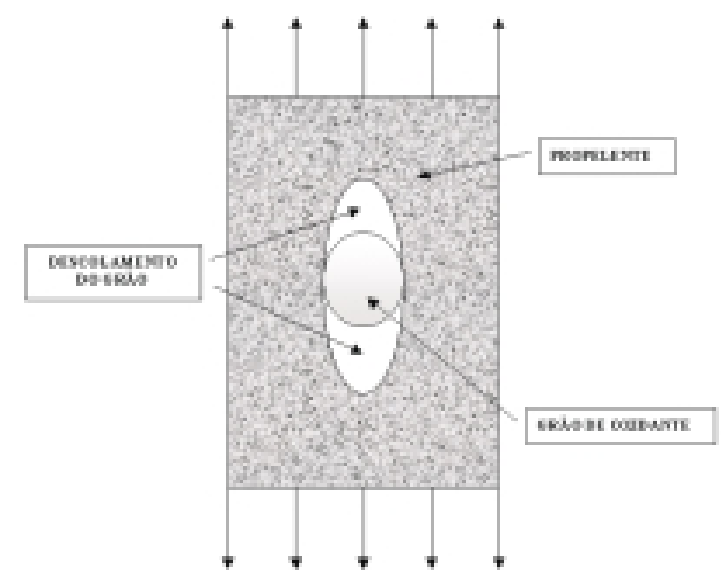

Figura 2. Grão da partícula oxidante descolada da matriz polimérica em um propelente tensionado, sem agente de ligação

Para evitar estes efeitos e promover a interação química e física entre a parte polimérica e os sólidos, são adicionados agentes de ligação. Estes são compostos que evitam a separação entre o polímero e as partículas sólidas, conferindo melhor resistência 'a umidade, melhores características de envelhecimento, maior resistência à tração e ao alongamento, eliminando a fragilidade mecânica do propelente, tornando-o menos quebradiço ${ }^{16,17}$, resultando em um propelente onde todos os componentes constituintes estão intimamente ligados de modo que, idealmente, formam um só corpo.

O agente de ligação é considerado na indústria de propelentes como produto estratégico e sua síntese e as propriedades atribuídas por ele ao propelente são guardadas como segredo industrial. Poucos trabalhos sobre o assunto são publicados e quando são, fazemno de maneira mais genérica possível, evitando assim, descrever a fórmula do produto e método de obtenção, citando apenas os resultados obtidos em sua aplicação sem entrar em maiores detalhes. A maior parte dos trabalhos publicados está na forma de patentes e, nas mais recentes, a fórmula do agente de ligação é simplesmente substituida por asteriscos ${ }^{18,19}$. A razão de tanto segredo é a importância e influência do agente de ligação no propelente.

$\mathrm{O}$ agente de ligação tem a capacidade de melhorar, e muito, as propriedades mecânicas do propelente ${ }^{16,20}$, principalmente as propriedades conhecidas como resistência à tração na ruptura e alongamento. De acordo com a Figura 3 e a Tabela 1, pode ser verificado como pequenas quantidades destes compostos melhoram significantemente as propriedades mecânicas do propelente ${ }^{11}$.

$\mathrm{O}$ agente de ligação promove a interação entre a carga sólida (perclorato de amônio e alumínio) e a matriz polimérica (binder). São compostos com funções orgânicas de amidas aziridínicas hidroxiladas ou não, aminas di ou trihidroxiladas, aminas cianohidroxiladas, amidas di hidroxiladas entre outras. Os agentes de ligação atuam sobre os sólidos, perclorato de amônio e alumínio, através de ligações verdadeiras e/ou por ligações secundárias, adsorção ou atração de cargas ${ }^{21,23}$. Atuam sobre o binder através de ligações verdadeiras ${ }^{24}$, reagindo, no caso de propelentes poliuretânicos com os grupos isocianatos e hidroxilas ${ }^{17,21,24}$, de modo que as cargas sólidas e a matriz polimérica estejam intimamente associadas, formando um corpo sólido com aspecto o mais homogênio possível. Wallace II $^{17}$ relata que a função amina do agente de ligação reage com o grupo isocianato do binder. Hori $^{21}$ afirma que a função aziridina do agente de ligação reage com a hidroxila do binder e $\mathrm{Nema}^{24}$ relata que a hidroxila do agente de ligação reage com o grupo isocianato do binder.

Ele, agente de ligação, deve envolver os sólidos cobrindo toda a 


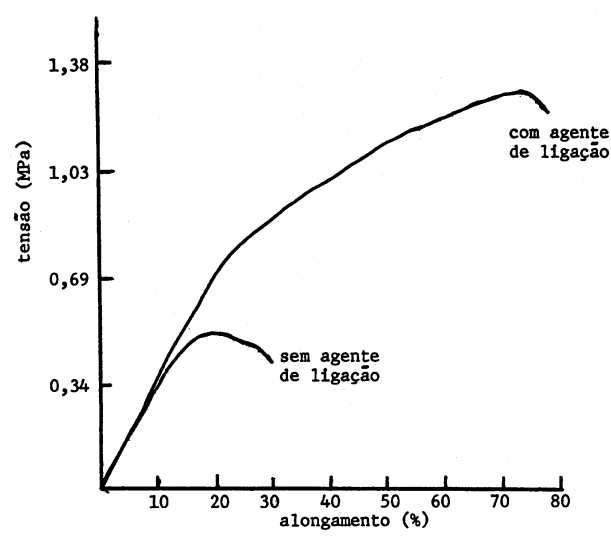

Figura 3. Efeito produzido por uma massa de agente de ligação equivalente a $0,35 \%$ da massa total do propelente à temperatura de $25{ }^{\circ} \mathrm{C}$.

Tabela 1. Efeito do agente de ligação nas propriedades mecânicas do propelente.

\begin{tabular}{cccc}
\hline $\begin{array}{c}\text { Temperatura } \\
\left({ }^{\circ} \mathrm{C}\right)\end{array}$ & $\begin{array}{c}\text { Agente de } \\
\text { ligação }\end{array}$ & $\begin{array}{c}\text { Tensão } \\
\text { máxima (Mpa) }\end{array}$ & $\begin{array}{c}\text { Alongamento na } \\
\text { tensão máxima }(\%)\end{array}$ \\
\hline 82 & Com & 0,59 & 26 \\
& Sem & 0,42 & 15 \\
25 & Com & 1,27 & 75 \\
& Sem & 0,52 & 19 \\
0 & Com & 1,96 & 89 \\
& Sem & 1,24 & 22 \\
-40 & Com & 2,89 & 72 \\
& Sem & 1,90 & 21 \\
-60 & Com & 5,65 & 35 \\
& Sem & 4,20 & 14 \\
\hline
\end{tabular}

superfície da partícula para formar uma camada firme e resistente sobre os mesmos, envolvendo-os completamente permitindo que se liguem à matriz polimérica ${ }^{25-27}$. A adsorção ocorre normalmente devido à presença de grupos polares na molécula do agente de ligação $0^{27}$ enquanto grupos aziridínicos homopolimerizam sobre os sólidos, devido a abertura do anel ${ }^{28}$.

Os agentes de ligação com estrutura de aminas e amidas atuam sobre o perclorato de amônio preferencialmente através de ligações secundárias ${ }^{21}$.

Convêm lembrar que o grão de alumínio possui uma superfície irregular o que também facilita a sua interação com a matriz elastomérica $^{13,29}$.

Portanto, de uma maneira geral, as seguintes especificações são requeridas de um agente de ligação $0^{5,11}$ :

- Ser bi ou trifuncional, para que ocorra a sua conversão.

- Interagir quimicamente com a fase orgânica do propelente

- Interagir física e/ou quimicamente com a carga sólida do propelente

- Ter estabilidade térmica e química

Acredita-se que o agente de ligação deva ser dissolvido na massa do propelente e, que permaneça dissolvido até mesmo após a adição dos sólidos. Publicações encontradas na literatura ${ }^{30}$ relatam que compósitos, com melhores propriedades mecânicas e até mesmo melhores características de envelhecimento, são mais facilmente conseguidos com agentes de ligação apenas miscíveis do que com agentes de ligação solúveis na massa elastomérica. Supõe-se que um agente de ligação miscível, portanto pouco solúvel, dirige-se para a superfície do oxidante cobrindo-o e posteriormente reage com os componentes orgânicos do propelente, especialmente o agente de cura. $\mathrm{O}$ perclorato de amônio, assim encapsulado, fica mecânica e quimicamente colado à matriz polimérica.
Por outro lado, se o agente de ligação é solúvel na massa, ele pode reagir quimicamente com os outros ingredientes presentes, especialmente a resina e o agente de cura, antes de cobrir a superfície do grão de perclorato de amônio. Se a superfície do oxidante não está adequadamente coberta pelo agente de ligação, o perclorato de amônio está apenas parcialmente ligado à matriz polimérica, o que resulta em propriedades mecânicas abaixo do esperado.

A importância do agente de ligação, portanto, está na sua função de fortalecer a ligação dos sólidos, especialmente do oxidante, com a matriz polimérica, evitando a propagação de fendas no propelente e melhorando as suas propriedades mecânicas ${ }^{31}$.

\section{AGRADECIMENTOS}

À Laís Tereza Fabri, Ronaldo Honório Marciano e Luiz Gonzaga Moreira pela valiosa colaboração na obtenção das figuras que ilustram o artigo.

\section{REFERÊNCIAS}

1. Klager, K.; Wrightson, J. M.; In Mechanics and Chemistry of Solid Propellants; Eringen, A. C. et al, Ed.; Pergamon Press; Oxford, 1967; p. 47.

2. Kubota, N. In Progress in Astronautics and Aeronautics - Fundamentals of Solid-Propellant Combustion; Kuo, K.K.; Summerfield, M., Ed.; AIAA, New York, 1984, 90, p. 1.

3. Price, E. W. In Progress in Astronautics and Aeronautics - Fundamentals of Solid-Propellant Combustion; Kuo, K.K.; Summerfield, M., Ed.; AIAA, New York, 1984, 90, p. 479.

4. da Silva, M. O.; Relatório Interno Doc. 043-EDQ-T/CTA, 1976, p. 5

5. Davenas, Alain. In Solid Rocket Propulsion Technology.; Davenas, A., Ed.; Pergamon Press, London, 1993, p. 415.

6. Murray, S. C. In Advanced Propellants Chemistry; Gould, R. F., Ed.; Washington D. C., 1966, p. 93.

7. Kishore, K.; Gayathri, V. In Progress in Astronautics and Aeronautics Fundamentals of Solid-Propellant Combustion; Kuo, K.K.and Summerfield, M., Ed.; AIAA, New York, 1984, 90, p. 68.

8. Al-Harthi, A.; Willians, A.; Fuel 1998, 77, 1451.

9. Bruenner, R. S.; Oberth, A. E.; J. Org. Chem. 1966, 31, 887.

10. Oertel, Günter; Polyurethane Handbook: Chemistry - Raw Materials Processing Application - Properties, 2. ed., Munich, Hanser Publishers, 1993, p. 13.

11. Oberth, A. E.; Bruenner, R. S. In Propellants, Manufacture, Hazards and Testing - Advanced Chemistry Series 88, Gould, R. F., Ed.; Washington D. C., 1969 , p. 84

12. Stutchbury, J. E.; Polymer as Fuel-binders for Solid Rocket Propellants. Proceedings of the Royal Australian Chemical Institute 1970, 37, 145.

13. Consaga, J. P.; U.S. Patents 4,214,928 1980.

14. Consaga, J. P.; U.S. Patents 4,944,815 1990.

15. Bruenner, R. S.; Oberth, A. E.; Trans. Soc. Rheology 1965, 9, 165.

16. Klager, K.; 20th Joint Propulsion Conference, Cincinnati, Ohio, 1984.

17. Wallace II, I. A.; U.S. Patents 5,472,532 1995.

18. Monte, S. J.; U.S. Patents 5,753,853 1998.

19. Hamilton, R.S.; U.S. Patents 5,336,343 1994.

20. Kumar, R. N; Cullick, F. E. C.; Combust. Sci. Technol. 1973, 8, 149.

21. Hori, K.; Iwama, A.; Fukuda, T.; Propellants, Explos., Pyrotech. 1990, 15,99

22. Hori, K.; Iwama, A.; Fukuda, T.; $18^{\text {th }}$ International Jahrestag - Fraunhofer Inst. Treib, Explosivst, Karlsruhe, Alemanha, 1987.

23. Hori, K.; Iwama, A.; Fukuda, T.; Propellants, Explos., Pyrotech. 1985, $10,176$.

24. Nema, S. K.; Nair, P. R.; Francie, A. U.; Gowariker, V. R.; AIAA Papers, 1977, 77, 932.

25. Bayley, A.; Ballerby, J. M.; Kinlock, S. A.; Philosof. Trans. Royal Soc. London 1992, 339, 321.

26. Bruenner, R. S.; U.S. Patents 4,410,376 1983.

27. Klager, K; Di Millo, A. J. In Encyclopedia of Polymer Science and Technology, Bikales, N., Ed.; New York, 1970, 12, 105.

28. Oberth, A. E.; U.S. Patents 5,477,895 1995.

29. Hendrickson, R. R.; et al, U.S. Patents 5,859,384 1999.

30. Ducote, M. E.; U.S. Patents 4,531,989 1985.

31. Conyers, J. A.; U.S. Patents 4,332,632 1982. 